\title{
SOME APPLICATIONS OF HEWITT'S FACTORIZATION THEOREM
}

\author{
H. S. COLLINS AND W. H. SUMMERS
}

1. Introduction. In the last ten years several papers have appeared in the literature which (somewhat in retrospect!) deal with Banach algebras $A$ with bounded approximate identity which act on some Banach space $V$, the usual requirements being that $V$ be at least a left $A$-module and the approximate identity act as a left approximate identity for $V$. In this paper we follow Hewitt [9] in making the minimal restriction that the bounded approximate identity be only a left one for both $A$ and $V$.

Our main contribution to this circle of ideas is in the presentation (in \$2) of a device apparently unobserved until now; namely, the introduction of an auxiliary left $A$-module which arises in a natural way. This device, in conjunction with Hewitt's factorization theorem [9, p. 151], yields (in \$3) many and diverse results, some of them new while others are significant extensions of known results. We do not believe that all the possibilities of this procedure are exhausted here; indeed, it is expected that further applications will be found, as well as extensions to actions of Banach algebras with approximate identity on more general locally convex spaces (for example, to the weighted locally convex spaces considered in [13]).

2. The space $m(\Lambda ; V ; ß)$. From now on $V$ will denote a complex Banach space, $\Lambda$ a set, $A$ a complex Banach algebra, and $B$ a collection of bounded subsets of $V$ which satisfies one or more of the following three properties:

$(\alpha) Q B$ is closed under addition.

$(\beta) B$ is closed under multiplication by complex scalars.

$(\gamma)$ A subset $B$ of $V$ is in $B$ whenever for each $\epsilon>0$ there is a $B_{\epsilon} \in B$ such that $B$ is contained in the union of the open balls of radius $\epsilon$ centered at the points of $B_{\mathbf{\epsilon}}$ (i.e., $B_{\mathbf{e}}$ is an $\epsilon$-net for $B$ ).

The following additional property which is encountered when $V$ is a left $A$-module and $A$ has a bounded left approximate identity $\left\{a_{j}\right\}$ which acts as a left approximate identity for $V$ will also be needed:

$(\delta)\left\{a_{j}\right\}$ converges uniformly to the identity on each member of $B$

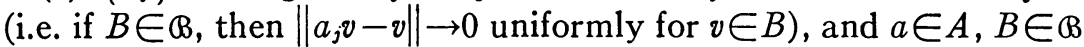
implies $a B \in B$.

Presented to the Society, October 26, 1968; received by the editors June 7, 1968. 
We now list several examples of collections $B$, almost all of which will be needed in the sequel.

$B_{0}$ : all bounded subsets of $V$;

$B_{1}$ : all totally bounded subsets of $V$;

$B_{2}$ : all $\beta$-equicontinuous subsets of $M(S)$ (here, and throughout the rest of this paper, $S$ will denote a locally compact Hausdorff space, $M(S)$ the variation normed Banach space of bounded Radon measures on $S$, while $\beta$ is the strict topology on the space $C(S)$ of bounded continuous complex functions on $S$ (see Buck [1]));

$B_{3}$ : all subsets $B$ of $M(S)$ having the property that each sequence in $B$ has a $\sigma(M(S), C(S))$ cluster point in $M(S)$;

$B_{4}$ : all countably $\beta$-equicontinuous subsets of $M(S)$ (i.e., $B \in \bigotimes_{4}$ if and only if each sequence in $B$ is $\beta$-equicontinuous);

$B_{5}$ : (here we assume $A$ has a bounded left approximate identity $\left\{a_{j}\right\}$ which acts as a left approximate identity on the left $A$-module $V)$ all bounded subsets of $V$ on which $\left\{a_{j}\right\}$ converges uniformly to the identity.

2.1. REMARK. One routinely verifies that $B_{0}, B_{1}, B_{2}, B_{4}$, and $B_{5}$ all satisfy both $(\alpha)$ and $(\beta)$, and that $B_{0}$ and $\beta_{5}$ satisfy $(\gamma)$. One uses [10 p. 42 ] to see that $B_{1}$ satisfies $(\gamma)$, and Conway's criterion for $\beta$-equicontinuity $[4$, p. 76$]$ leads to an easy proof that $B_{2}$ and $B_{4}$ satisfy $(\gamma)$. While $B_{3}$ satisfies $(\beta)$, it does not in general seem to satisfy either $(\alpha)$ or $(\gamma)$, but further comments later on (see Theorem 3.4) will help clarify the situation relative to $B_{3}$. We note here that $B_{0}$ does not in general satisfy $(\delta)$, while $B_{1}$ and $B_{5}$ will always satisfy $(\delta)$.

We denote by $m(\Lambda ; V)$ the Banach space of all bounded functions on $\Lambda$ to $V$ with the usual pointwise operations and $\|f\|_{\infty}$ $=\sup \{\|f(\lambda)\|: \lambda \in \Lambda\}$, and by $m(\Lambda ; V, \Theta)$ the subset of $m(\Lambda ; V)$ consisting of those $f$ for which $f(\Lambda) \in B$. Since $(\gamma)$ implies that subsets of members of $B$ also belong to $B$, we see that $m(\Lambda ; V ; B)$ is a Banach space whenever $B$ satisfies $(\alpha),(\beta)$, and $(\gamma)$. We may now state the theorem which together with Hewitt's factorization theorem yields the results of $\$ 3$.

2.2. Theorem. Suppose $V$ is a left $A$-module and $\left\{a_{j}\right\}$ is a bounded left approximate identity for $A$ that acts on $V$ as a left approximate identity. If $B$ satisfies $(\alpha),(\beta),(\gamma)$, and $(\delta)$, then $m(\Lambda ; V ; \beta)$ is a Banach space which is a left $A$-module (under the pointwise action) and $\left\{a_{j}\right\}$ acts as a left approximate identity on $m(\Lambda ; V ; \otimes)$.

Proof. The conclusion follows from the remarks preceding the theorem and the assumption that $B$ satisfies $(\delta)$. 
3. Applications. In our first theorem we give necessary and sufficient conditions that $V$ have the metric approximation property [8, I, p. 178] (hereafter abbreviated to m.a.p.), and then in the second theorem we extend (and generalize) to all such spaces a result of Raimi [11, p. 643] proved only for Hilbert spaces. According to Grothendieck [8, I, p. 182], all known spaces $V$ have m.a.p. (including all separable Banach spaces with a Schauder basis). Our next results yield two apparently hitherto unnoticed facts concerning the $\beta$ topology on $C(S)$, and give yet another characterization of those $C(S)_{\beta}(C(S)$ with the $\beta$-topology) which are semireflexive (see $[5$, p. 477]). It seems likely that techniques similar to these may give a simple proof of Conway's theorem $\left[4\right.$, p. 76] that $C(S)_{\beta}$ is a strong Mackey space when $S$ is paracompact. Finally, we give simple proofs (and extensions) of certain results found in [14] and [12], one being a characterization of those Banach algebras with bounded left approximate identity, while another is a similar result needed by the authors in [12] for their work on a general approach to strict topologies. We now proceed to the theorems.

3.1. Theorem. $V$ has m.a.p. if and only if the Banach algebra $A$ (operator norm) of compact operators on $V$ contains a bounded left approximate identity $\left\{a_{j}\right\}$ consisting of operators of finite rank which also acts as a left approximate identity for $V$.

Proof. To see the "if" part, we let $A$ act on $V$ in the obvious way and take $\Lambda=V$. If $Z \in \beta_{1}$ and if $f$ is defined on $\Lambda$ to $V$ to be the identity on $Z$ and zero off $Z$, then $f \in m\left(\Lambda ; V ; \beta_{1}\right)$. It follows from Remark 2.1 and Theorem 2.2 that $\left\{a_{j}\right\}$ acts as a left approximate identity on $m\left(\Lambda ; V ; Q_{1}\right)$, i.e. $\left\|a_{j} f-f\right\|_{\infty} \rightarrow 0$, which implies $\left\{a_{j}\right\}$ converges uniformly to the identity on $Z$, and thus $V$ has m.a.p.

For the converse, there is a net $\left\{a_{j}\right\}$ in $A$ which is a bounded set of finite rank operators and which converges to the identity uniformly on members of $\beta_{1}$. If $a \in A$, then

$$
\left\|a_{j} a-a\right\|=\sup \left\{\left\|a_{j} a v-a v\right\|:\|v\| \leqq 1\right\} \underset{j}{\rightarrow} 0
$$

since $\{a v:\|v\| \leqq 1\} \in B_{1}$, i.e. $\left\{a_{j}\right\}$ is a bounded left approximate identity for $A$. To see that this is also true for $V$, choose $f$ in the dual $V^{*}$ of $V$ so that $f(v)=1$ where $v \in V$. Then $f \otimes v \in A$ and $f \otimes v(v)=f(v) v=v$ from whence it follows that $a_{j} v \rightarrow v$.

3.2. THEOREM. If $V$ has m.a.p., then for any $Z \in \beta_{1}$ there is a compact operator $a$ on $V$ and $a Y \in B_{1}$ such that $Z=a Y$. 
Proof. Note that if $U$ is the closed unit ball in $V$, then there is a $c>0$ so that $Y \subseteq c U$ and hence Theorem 3.2 would yield $a Y$ $=Z \subseteq a(c U)=(c a) U$ which is the exact statement of Raimi's result for Hilbert spaces [11, p. 643].

Now let $A$ be the compact operators on $V$, let $\Lambda=V$, and let $f$ be defined on $\Lambda$ to $V$ to be the identity on $Z$ and zero off $Z$. Then $f \in m\left(\Lambda ; V ; \bigotimes_{1}\right)$, and it follows from Theorem 3.1 (in conjunction with Theorem 2.2$)$ that $A$ and $m\left(\Lambda ; V ; \bigotimes_{1}\right)$ satisfy the hypothesis of Hewitt's factorization theorem. Hence there is a $g \in m\left(\Lambda ; V ; B_{1}\right)$ and $a \in A$ such that $f=a g$. If we let $Y=\{g(\lambda): \lambda \in Z\}$, then $Y \in B_{1}$ and $Z=a Y$.

We can at this time use our methods to give a new characterization of the $\beta$-equicontinuous subsets of $M(S)$ (Theorem 3.4.(1) below). Other characterizations have been given much earlier by Glicksberg [7] and later by Conway [4]. We also include a simple proof of the factorization which formed the basis of Buck's proof [1] that the dual of $C(S)_{\beta}$ is $M(S)$, show that each (norm) totally bounded set in $M(S)$ is $\beta$-equicontinuous (hitherto unobserved), and give a new description (in terms of $\beta$-equicontinuous sets) of those $S$ for which $C(S)_{\beta}$ is semireflexive.

3.3. REMARK. The space $C_{0}(S)$ of all continuous complex functions on $S$ which vanish at infinity (with the supremum norm) is a Banach algebra with a (bounded) canonical approximate identity (see [3, p. 158]) $\left\{a_{j}\right\}$, which acts on $M(S)$ in a natural way, i.e. if $a \in C_{0}(S)$ and $v \in M(S)$, then $a v(E)=\int_{E} a d v$ where $E$ is any Borel set in $S$. It is easy to check that $\left\{a_{j}\right\}$ acts as a left approximate identity on $M(S)$. A direct application of Hewitt's factorization theorem shows that if $v \in M(S)$, then there is a $u \in M(S)$ and $a \in C_{0}(S)$ so that $v=a u$.

3.4. Theorem. If $A=C_{0}(S),\left\{a_{j}\right\}$ is a canonical approximate identity in $A, V=M(S)$, and $\Lambda=V$, then

(1) $B_{1} \subseteq B_{2} \subseteq B_{4} \subseteq B_{3}$ and $B_{2}=B_{5}$ (thus $B_{2}$ satisfies $(\delta)$ ).

(2) The following are equivalent:

(i) $B_{1}=B_{2}$;

(ii) $C(S)_{\beta}$ is semireflexive;

(iii) $S$ is discrete.

(3) The following are equivalent:

(i) $B_{2}=B_{3}$;

(ii) $C(S)_{\beta}$ is a strong Mackey space;

(iii) $m\left(\Lambda ; V ; B_{3}\right)$ is a Banach space and a left $A$-module on which $\left\{a_{j}\right\}$ acts as a left approximate identity.

Proof. That $B_{2} \subseteq B_{4} \subseteq B_{3}$ is immediate from their definition. Using 
Remark 2.1, Theorem 2.2, and Remark 3.3, and Hewitt's factorization theorem, we see that if $B \in Q_{1}$, then there is an $a \in A$ so that $B \subseteq a U$ where $U$ is the closed unit ball in $V$. It now follows from the Glicksberg-Conway characterization of members of $B_{2}$ (e.g., see $\left[4\right.$, p. 76]) that $B \in B_{2}$. We remark that Conway's alternate criterion (used in Remark 2.1) can also be used to show $B_{1} \subseteq B_{2}$, but this seems to have been overlooked. Another application of the GlicksbergConway result for members of $B_{2}$ shows that $B_{2} \subseteq B_{5}$. We also have from Theorem 2.2 and Hewitt's factorization theorem that if $B \in B_{5}$, then there is a $C \in B_{5}$ and $a \in A$ so that $a C=B$. Again, using the Glicksberg-Conway criterion for $\beta$-equicontinuity, together with the fact that $C$ is (norm) bounded, we have that $B \in B_{2}$. This completes the proof of (1).

For the proof of (2), we note that the equivalence of (ii) and (iii) was shown in $[\mathbf{5}$, p. 477], while (iii) implies (i) may be found in [2, Theorem 4.1]. If $S$ is not discrete, then there is a sequence $\left\{x_{n}\right\}$ in $S$ contained in a compact set with $x_{n} \neq x_{m}, m \neq n$. By Urysohn's lemma, there is an $a \in A$ such that $a\left(x_{n}\right)=1$ for each $n=1,2, \cdots$, and so $\left\{\delta\left(x_{n}\right): n \geqq 1\right\}$, where $\delta(p)$ is the point mass at $p \in S$, is contained in the $\beta$-equicontinuous set $a U$ ( $U$ being the closed unit ball in $M(S)$ ). Since $\left\|\delta\left(x_{n}\right)-\delta\left(x_{m}\right)\right\|=2$ when $n \neq m$, we have $\beta_{1} \neq B_{2}$.

To see (3), we first note that the equivalence of (i) and (ii) is merely the definition of strong Mackey. Since $\Theta_{2}$ satisfies ( $\delta$ ) (from (1)), it follows from Remark 2.1 and Theorem 2.2 that (i) implies (iii). If (iii) holds, then for each $B \in \mathbb{B}_{3}$ an application of Hewitt's factorization theorem yields a $C \in \mathbb{B}_{3}$ and $a \in A$ such that $B=a C$. Hence $B \in B_{2}$, and we have from (1) that (i) holds, concluding the proof.

The last three theorems of this paper (which we prove for arbitrary Banach algebras with bounded left approximate identity) were originally proved for commutative Banach algebras with bounded approximate identity, and thus represent significant extensions.

3.5. Theorem (TAylor [14]). Suppose $C \geqq 1$. The following are equivalent:

(1) $Z$ totally bounded in $A$ and $\delta>0$ imply there exists $a \in A,\|a\| \leqq C$ and $a$ totally bounded $Y$ in $A$ so that (1a) $Z=a Y$, (1b) $\left\|a a^{\prime}-a^{\prime}\right\|<\delta$, $a^{\prime} \in Y,(1 \mathrm{c}) Y$ is contained in the closed ideal generated by $Z$, and (1d) $a^{\prime} \rightarrow a a^{\prime}$ is a homeomorphism of $Y$ onto $Z$.

(2) $Z$ finite in $A, \delta>0$ imply, there exists $a \in A,\|a\| \leqq C$, and $Y \subseteq A$ such that (2a) $Z=a Y$, (2b) $\left\|a a^{\prime}-a^{\prime}\right\|<\delta, a^{\prime} \in Y$.

(3) $A$ has a left approximate identity bounded by $C$.

Proof. Our only contribution is an application of our methods to 
a proof of $(3) \Rightarrow(1)$; the other implications are as in Taylor's paper [14]. Thus, we assume (3) holds, and fix $Z$ totally bounded, $\delta>0$. Setting $\Lambda=A=V$ and $B=B_{1}$ in our Theorem 2.2, we see that Hewitt's factorization theorem applies (for the first time we invoke its full strength), and defining $f$ on $\Lambda$ to $V$ to be the identity on $Z$ and zero off $Z$ (hence $f \in m\left(\Lambda ; V ; \cap_{1}\right)$ ), there exists $a \in A$ and $g \in m\left(\Lambda ; V ; \bigotimes_{1}\right)$ so that $f=a g, g \in \operatorname{cl}(A f)$ (closure in $\left.m\left(\Lambda ; V, B_{1}\right)\right),\|f-g\|_{\infty}<\delta$, and $\|a\| \leqq C$. It then follows that $Z=a Y$ where $Y=\{g(\lambda): \lambda \in Z\}$, and if $a^{\prime} \in Y$, then $\left\|a a^{\prime}-a^{\prime}\right\|=\|a g(\lambda)-g(\lambda)\| \leqq\|f-g\|_{\infty}<\delta$. To obtain (1c), note there is a sequence $\left\{a_{n}\right\}$ in $A$ so that $\left\|a_{n} f-g\right\|_{\infty} \rightarrow 0$; if $a^{\prime} \in Y$, then $a^{\prime}=g(\lambda)$ with $\lambda \in Z$ and $\left\|a_{n} f(\lambda)-g(\lambda)\right\| \leqq\left\|a_{n} f-g\right\|_{\infty} \rightarrow 0$ (i.e., $a^{\prime} \in \operatorname{cl}(A f(\lambda)$ ) (closure in $A)$ ). To conclude the proof we need verify $(1 \mathrm{~d})$, and this is straightforward.

The following result due to Curtis and Figá-Talamanca follows directly from Hewitt's factorization theorem.

3.6. Theorem [6, p. 174]. Let $A$ be an algebra of bounded linear operators on a Banach space $X$ and suppose under some norm stronger than the operator norm that $A$ is a Banach algebra with bounded left approximate identity $\left\{a_{j}\right\}$. If $A \cdot X=\left\{v \in X: a_{j} v \rightarrow v\right\}$ and if $A X$ $=\{a v: a \in A, v \in X\}$, then $A \cdot X=A X$.

Proof. The equation $a_{j} v-v=a_{j}\left(v-v_{\alpha}\right)+\left(a_{j} v_{\alpha}-v_{\alpha}\right)+\left(v_{\alpha}-v\right)$ shows $A \cdot X$ is a Banach subspace of $X$, which is a left $A$-module on which $\left\{a_{j}\right\}$ acts as a left approximate identity. Certainly $A X \subseteq A \cdot X$, while Hewitt's factorization theorem shows $A \cdot X \subseteq A X$.

3.7. Theorem (Sentilles and Taylor [12]). Let $A$ and $X$ be as in Theorem 3.6, suppose $\left\{a_{j}\right\}$ is bounded by $C \geqq 1$ and $Z \subseteq A \cdot X$. Then the following are equivalent:

(1) There exists $a \in A$ and bounded $W \subseteq A \cdot X$ so that $a W=Z$.

(2) $Z$ is bounded and $\left\{a_{j}\right\}$ converges uniformly to the identity on $Z$.

(3) For each $\delta>0$ there exists $a \in A,\|a\| \leqq C$, and bounded $W \subseteq A \cdot X$ so that (a) $Z=a W$, (b) $\|a w-w\|<\delta$, weW, and (c) $a$ is a homeomorphism of $W$ onto $Z$.

Proof. We check only (2) $\rightarrow(3)$, and as usual by the use of Theorem 2.2. For $\Lambda=A \cdot X=V$, an application of Hewitt's theorem to $m\left(\Lambda ; V ; B_{5}\right)$ as an $A$-module concludes the proof.

\section{References}

1. R. C. Buck, Bounded continuous functions on a locally compact space, Michigan Math. J. 5 (1958), 95-104.

2. H. S. Collins, On the space $l^{\infty}(S)$, with the strict topology, Math. Z. 106 (1968), 361-373. 
3. H. S. Collins and J. R. Dorroh, Remarks on certain function spaces, Math. Ann. 176 (1968), 157-168.

4. J. B. Conway, The strict topology and compactness in the space of measures, Bull. Amer. Math. Soc. 72 (1966), 75-78.

5. - The strict topology and compactness in the space of measures, Trans. Amer. Math. Soc. 126 (1967), 474-486.

6. P. Curtis and A. Figa-Talamanca, "Factorization theorems for Banach algebras" in Function algebras, edited by F. J. Birtel, Scott Foresman and Co., Chicago, Ill., 1966, pp. 169-185.

7. I. Glicksberg, Bishop's generalized Stone-Weierstrass theorem for the strict topology, Proc. Amer. Math. Soc. 14 (1963), 329-333.

8. A. Grothendieck, Produits tensoriels topologiques et espaces nucleaires, Mem. Amer. Math. Soc. No. 16 (1955).

9. E. Hewitt, The ranges of certain convolution operators, Math. Scand. 15 (1964), 147-155.

10. M. A. Naimark, Normed rings, Noordhoff, Groningen, 1964.

11. R. A. Raimi, Compact transformations and the k-topology in Hilbert space, Proc. Amer. Math. Soc. 6 (1955), 643-646.

12. D. Sentilles and D. Taylor, Factorization in Banach algebras and the general strict topology, Trans. Amer. Math. Soc. 141 (1969).

13. W. H. Summers, Weighted locally convex spaces of continuous functions, Ph.D. Dissertation, Louisiana State University, 1968.

14. D. Taylor, A characterization of Banach algebras with approximate unit, Bull. Amer. Math. Soc. 74 (1968), 761-766.

Louisiana State University 